\title{
ENVOLVIMENTO DO CONSUMIDOR EM PROCESSOS DE DESENVOLVIMENTO DE PRODUTOS: UM ESTUDO QUALITATIVO JUNTO A EMPRESAS DE BENS DE CONSUMO
}

INVOLVEMENT OF THE CONSUMER IN THE PRODUCT DEVELOPMENT PROCESSES: A QUALITATIVE STUDY OF CONSUMER GOODS COMPANIES

PARTICIPACIÓN DEL CONSUMIDOR EN PROCESOS DE DESARROLLO DE PRODUCTOS: UN ESTUDIO CUALITATIVO CON EMPRESAS DE BIENES DE CONSUMO

\section{RESUMO}

Na última década, emergiu na literatura de Marketing a discussão sobre coprodução de valor, destacando-se o envolvimento de consumidores nos processos de desenvolvimento de produtos (PDP), conceito associado à interação e inserção de consumidores nos processos de criação de valor. O presente estudo foi realizado por meio de análise de conteúdo com empresas brasileiras de bens de consumo para examinar o envolvimento de consumidores nos PDP. Apesar de as empresas apresentarem características de inovação e de sucesso em novos produtos, o envolvimento dos consumidores nos PDP ocorre com intensidade variada e a partir de mecanismos bastante distintos. Nos resultados são caracterizados os mecanismos de envolvimento, os estágios dos PDP em que o envolvimento ocorre e os níveis de intensidade de envolvimento dos consumidores. Finalmente, são apresentadas as implicações gerenciais e acadêmicas do estudo, seguidas de suas limitações, e recomendações para estudos futuros.

PALAVRAS-CHAVE Envolvimento de consumidores, desenvolvimento de produtos, coprodução de valor, cocriação, marketing de bens de consumo.

\section{Carolina Rosado dos Santos carolrosado@hotmail.com}

Gerente de Planejamento e Informações de Marketing, Universidade do Vale do Rio dos Sinos - Porto Alegre - RS, Brasil

\section{Vinícius Sittoni Brasil vinicius@pucrs.br}

Coordenador do Programa de Pós-Graduação em Administração, Pontifícia Universidade Católica do Rio Grande do Sul - Porto Alegre - RS, Brasil

\section{Recebido em 23.11.2009. Aprovado em 29.07.2010}

Avaliado pelo sistema double blind review

Editor Científico: Eduardo André Teixeira Ayrosa

\begin{abstract}
Over the last decade a discussion has arisen in marketing literature about value co-production, which highlights the involvement of consumers in product development processes (PDP), a concept associated with the interaction and inclusion of consumers in value creation processes. This is a content analysis study, carried out with Brazilian consumer goods companies in order to investigate the involvement of consumers in PDP. Despite the fact that these companies are innovative and successful with their new products the involvement of consumers in the PDP occurs with varied degrees of intensity and starting from the quite different mechanisms. The results characterize the involvement mechanisms, stages of the PDP in which the involvement occurs and the intensity levels of such consumer involvement. Finally, the managerial and academic applications of the study are presented, followed by its limitations and recommendations for future studies.
\end{abstract}

KEYWORDS Consumer involvement, product development, value co-production, co-creation, consumer goods marketing.

RESUMEN En la última década, surgió en la literatura de Marketing la discusión sobre coproducción de valor, relacionada sobretodo a la participación de los consumidores en los procesos de desarrollo de productos (PDP), concepto asociado a la interacción e inserción de consumidores en los procesos de creación de valor. El presente estudio fue realizado por medio de análisis de contenido, con empresas brasileñas de bienes de consumo, para analizar la participación de los consumidores en el PDP. Si bien las empresas presentan características innovadoras y éxito en nuevos productos, la participación de los consumidores en el PDP ocurre con intensidad variada y a partir de mecanismos bastante distintos. En los resultados se caracterizan mecanismos de participación, etapas del PDP en que la participación ocurre y niveles de intensidad de participación de consumidores. Por fin se presentan implicaciones gerenciales y académicas del estudio, seguidas de limitaciones y recomendaciones para estudios futuros.

PALABRAS CLAVE Participación de consumidores, desarrollo de productos, coproducción de valor, cocreación, marketing de bienes de consumo. 


\section{INTRODUÇÃO}

O lançamento de novos produtos tem sido observado por acadêmicos e profissionais de Marketing como uma estratégia de empresas que buscam obter vantagens competitivas em relação aos seus concorrentes. Considerando-se a importância da estratégia de desenvolvimento de novos produtos apontada por Slater e Narver (1995) e Hamel e Prahalad (2004), investigar mecanismos e metodologias que contribuam para o sucesso dos novos produtos tem sido foco de questionamentos acadêmicos e gerenciais. Igualmente, cabe ressaltar os estudos sobre orientação para o mercado que sugerem que os produtos desenvolvidos com a orientação para os consumidores apresentam melhor desempenho no mercado (JAWORSKI; KOHLI, 1993; DAY; WENSLEY, 1988; NARVER; SLATER, 1990; DESHPANDE; FARLEY; WEBSTER, 1993; KUMAR; PETERSEN, 2005).

Slater e Narver (1995) já apontaram que as empresas que desejam desenvolver a sua capacidade de inovação devem buscar alternativas para complementar as técnicas tradicionais de pesquisa de mercado. Nesse contexto, o envolvimento de consumidores em processos de desenvolvimento de produtos (PDP) tem sido investigado como uma técnica alternativa para a coleta de informações valiosas para as empresas que buscam vantagens competitivas por meio do lançamento de produtos inovadores (VON HIPPEL, 1986; ALAM, 2002; NAMBISAN, 2002; URBAN; VON HIPPEL, 1988; LILIEN e outros, 2002 e FRANKE e outros, 2006).

Em termos gerenciais, grandes empresas como Adidas, Google, Skype, Procter \& Gamble, Peugeot, Volvo e Microsoft também estão operacionalizando interações com os seus consumidores durante o PDP. É necessário destacar que a interação com os consumidores tem sido impulsionada por novas ferramentas tecnológicas, como a internet, que apresenta recursos de interação a baixo custo para a empresa e com grande conveniência para os consumidores (NAMBISAN; BARON, 2007; MÖLLER e outros, 2008).

Este artigo apresenta como foco central o envolvimento de consumidores em processos de desenvolvimento de produtos, buscando analisar os estágios do PDP em que o envolvimento ocorre, os mecanismos de envolvimento de consumidores e os diferentes níveis de intensidade na interação empresa-consumidor. Ainda que estudos internacionais tenham abordado essa temática, verificase a ausência de trabalhos no contexto brasileiro que explorem especificamente os momentos e as formas de envolvimento de consumidores no PDP. Desse modo, o presente trabalho foi desenvolvido com a seguinte questão orientadora: como as empresas de bens de consumo brasileiras têm envolvido seus consumidores no PDP?

A fundamentação teórica está alicerçada na estrutura conceitual defendida por Lusch (2007), a qual divide a história do conceito de marketing em três perspectivas: "to market", "market(ing) to" e "market(ing) with". A relação dessas três perspectivas conceituais com o envolvimento de consumidores em processos de desenvolvimento de produtos é a ênfase do presente estudo. Também são discutidos modelos de desenvolvimento de produtos com o objetivo de fornecer a sustentação teórica sobre os estágios que permitem o envolvimento de consumidores.

\section{REFERENCIAL TEÓRICO}

O envolvimento de consumidores no PDP tem sido investigado como uma técnica alternativa para a coleta de informações para empresas que buscam vantagens competitivas por meio do lançamento de produtos inovadores (URBAN; VON HIPPEL, 1988; ALAM, 2002; FRANKE e outros, 2006).

É importante esclarecer inicialmente que o conceito de coprodução de valor aqui utilizado é o conceito defendido por Vargo e Lusch (2004). A coprodução pressupõe o envolvimento do consumidor na criação da essência da oferta e permite que consumidores e empresas compartilhem ideias de inovação, design ou novos produtos. Em relação ao conceito de envolvimento, é fundamental delimitar o distanciamento do conceito utilizado neste estudo do significado de envolvimento utilizado tradicionalmente na área de comportamento do consumidor. Nessa área, o envolvimento apresenta o sentido de relevância pessoal de um produto ou serviço (ENGEL; BLACWELL; MINIARD, 2000); enquanto no presente trabalho o conceito de envolvimento possui o significado de inserção, interação e engajamento de consumidores nos processos de coprodução de valor (GALLOUJ; WEINSTEIN, 1997; VARGO; LUSCH, 2004).

A literatura discute três escolas de pensamento que defendem três visões diferentes sobre o PDP: processo linear, processo recursivo e processo caótico (MCCARTHY e outros, 2006). O processo linear representa a visão tradicional do PDP, em que todos os seus estágios são conectados de forma seriada ou sequencial. A eficiência do projeto organizado de forma linear depende da cooperação, coordenação e comunicação das equipes multidisciplinares envolvidas no seu desenvolvimento. Diante das críticas aos modelos de processos lineares, considerados pouco 
ágeis e com maior demanda de tempo, são indicados os processos recursivos. Essa proposta permite que diferentes estágios sejam realizados de forma simultânea ou paralela, e que no decorrer do processo ocorram atividades de feedback com possibilidade de retorno aos estágios anteriores (MCCARTHY e outros, 2006; LEONARDBARTON, 1995). Por fim, em mercados em que a exigência de lançamento de produtos totalmente novos é elevada, MCCARTHY e outros (2006) recomendam a utilização do modelo caótico de desenvolvimento de produtos. O "caos" proposto está fundamentado na possibilidade da realização de estágios e atividades do processo de forma desordenada, objetivando que novas ideias surjam no decorrer do projeto, proporcionando o desenvolvimento de um produto potencialmente inovador.

Especificamente sobre o envolvimento de consumidores no PDP, foi possível identificar que a maior participação dos consumidores passava também por uma compreensão sobre conceitos fundamentais da área de Marketing. Para isso, este trabalho baseou sua fundamentação nos estudos liderados por Vargo e Lusch (2004), Lusch e Vargo (2006) e Lusch (2007) sobre a necessidade da criação de uma nova perspectiva conceitual para a área de Marketing.

Diante dessa escolha de literatura, a fundamentação teórica a seguir está dividida em três subseções que identificam a relação das perspectivas históricas do conceito de marketing com o envolvimento de consumidores no desenvolvimento de produtos: perspectiva "to market", perspectiva "market(ing) to" e perspectiva "market(ing) with".

\section{Perspectiva "to market"}

As primeiras conceituações sobre marketing expressaram durante cinco décadas a visão "to market", influenciando publicações e principalmente as discussões iniciais promovidas pela American Marketing Association. A essência dos primeiros conceitos está no papel do marketing como a interface entre o produtor e o comprador (LUSCH, 2007). Ao analisar as primeiras publicações, foram encontrados como temas mais frequentes: troca (vendas e compras), distribuição física (estoque e transporte) e funções financeiras (financiamento e análise de risco). Nos estudos pioneiros, até mesmo a palavra "marketing" era empregada como sinônimo de distribuição, trade e comércio (JONES, 2004; SHAW; JONES, 2005).

É importante ressaltar que, na perspectiva "to market," envolvimento, interação e preocupação com os consumidores não apresentam relevância na sua construção teórica e prática. As empresas identificadas com a perspectiva "to market" apresentam como principal função vender os bens físicos já produzidos. Identifica-se nesse contexto uma crença de que tudo o que as empresas produzem é consumido pelo mercado (JONES, 2004; SHAW; JONES, 2005; LUSCH, 2007).

$\mathrm{Na}$ perspectiva "to market" é possível observar que a interação de consumidores com as empresas estava restrita ao momento da compra, não apresentando, portanto, indícios de envolvimento de consumidores no PDP. Embora a abordagem "to market" tenha sido apresentada dentro de um contexto histórico sobre os primeiros conceitos de marketing, é possível encontrar empresas que ainda praticam tais fundamentos (LUSCH, 2007).

\section{Perspectiva "market(ing) to" e a visão de consumidor como recurso}

$\mathrm{Na}$ abordagem "market(ing) to", os primeiros estudos sobre comportamento do consumidor são apresentados e discutidos na academia. Trabalhos pioneiros como os de Engel e outros (1968) e Howard e Sheth (1969) tentaram compreender as motivações de compra dos consumidores. Dessa forma, a perspectiva "market(ing) to" começa a ser desenvolvida já com a preocupação do papel do consumidor nos processos de marketing (SHAW; JONES, 2005; LUSCH, 2007).

O conceito de marketing discutido na perspectiva "market(ing) to" relaciona diretamente as decisões gerenciais das organizações com o objetivo de satisfazer as necessidades dos consumidores. Ao apresentar a crítica da construção do marketing como um conjunto de processos que deveriam estar articulados para satisfazer as necessidades dos consumidores - e ao refletir sobre a dinâmica criada para que o consumidor seja considerado um recurso exógeno - Vargo e Lusch (2004) e Lusch (2007) apontam que o pensamento dominante nas últimas cinco décadas é o da perspectiva "market(ing) to".

Nessa perspectiva, estudos como Rothwell (1974), Von Hippel (1988), Leonard-Barton (1995) e Christensen (1997) apresentam a discussão sobre a utilização dos consumidores como um recurso dentro do processo de desenvolvimento de produtos. Leonard-Barton (1995), Cooper e Kleinschmidt (1995) e Christensen (1997) argumentam que o consumidor pode ser um recurso para a empresa, observando que o envolvimento de consumidores pode ser considerado uma fonte rica para o desenvolvimento de novos produtos, principalmente na fase de geração de ideias. Os autores ainda ressaltam que o envolvimento de consumidores tende a apresentar melhores resultados quando a empresa deseja realizar melhorias em produtos já existentes, ou seja, quando as empresas optam por realizar inovações do tipo "incremental". 
Na perspectiva "market(ing) to", a interação e o envolvimento com consumidores não estão vinculados à estratégia da empresa. O envolvimento, quando presente, ganha um caráter operacional dos processos de marketing, e o consumidor é visto somente como um recurso ou fonte de informações. Nesse sentido, não é possível reconhecer características de troca ou desenvolvimento do conhecimento do consumidor, tendo em vista que a transferência de conhecimento é apresentada como uma via de mão única, partindo do consumidor e terminando na empresa (VARGO; LUSCH, 2004; BALLANTYNE; VAREY, 2006).

Dentro da perspectiva de "market(ing) to", a geração de informações de mercado ocorre principalmente por meio de pesquisas surveys, grupos focais, observações de compra, entre outras técnicas tradicionais de coleta de dados (KAULIO, 1998; LILIEN e outros, 2002; MAKLAN e outros, 2007).

\section{Perspectiva "market(in) with" e os consumidores como coprodutores de valor}

A perspectiva denominada por Lusch (2007) "market(ing) with" difere das perspectivas "market(ing) to" e "to market" fundamentalmente no conceito de interação com os consumidores. Na abordagem "market(ing) with", o consumidor é considerado um parceiro que interage com a empresa e participa do processo de coprodução de valor. A interação do consumidor se dá pelo oferecimento das suas habilidades, competências e, principalmente, pelas suas experiências de consumo. Dessa forma, o consumidor é um agente ativo e endógeno no processo de marketing (LUSCH, 2007; PRAHALAD; RAMASWAMY, 2003, 2004).

Gallouj e Weinstein (1997) apresentaram o conceito de coprodução como uma forma de interação entre consumidores e empresas. No entanto, a discussão sobre a coprodução ganhou maior relevância com as proposições de Vargo e Lusch (2004). Os autores definem coprodução como o envolvimento e a participação do consumidor na criação da essência da oferta. Esse envolvimento pode ocorrer por meio do compartilhamento de ideias inovadoras, do desenvolvimento de design e de experiências (LUSCH, 2007).

Nesse contexto, o consumidor pode contribuir no núcleo de criação dos benefícios de um produto, e sua interação começa desde a geração de ideias até o efetivo consumo. É necessário também esclarecer que essa interação é compartilhada de forma integrada com outros atores (acionistas, consumidores, fornecedores, membros da comunidade e colaboradores da empresa). Forma-se, assim, uma rede de colaboração que permite as trocas necessárias para que o processo de interação se desenvolva e produza o valor necessário para consumidor e empresa (VARGO; LUSCH, 2004; LUSCH; VARGO, 2006; LUSCH, 2007).

Falando-se especificamente sobre os mecanismos de envolvimento dos consumidores, são encontradas desde técnicas tradicionais de pesquisa de marketing até sofisticadas tecnologias de interação. Entre as modalidades mais tradicionais de coleta de dados, Alam (2002) destaca as entrevistas de profundidade, as visitas e reuniões dos consumidores com a equipe de desenvolvimento de produto, a técnica de brainstorming com consumidores, a observação de consumidores, o recebimento de telefonemas, faxes, e-mails e a realização de grupos focais. Nambisan e Baron (2007) citam que algumas empresas se utilizam de recursos tecnológicos como fóruns de discussão na internet, kits de ferramentas que permitem a usuários criar design, protótipos virtuais e ainda jogos interativos que permitem simulações de utilização e a criação de novas funcionalidades.

Empresas que desejam potencializar o conhecimento do seu mercado têm investido na criação de meios interativos como as comunidades de prática. Estas proporcionam o aprendizado entre consumidores e empresas por meio da troca de ideias em grupo, da exposição de problemas comuns e da busca de soluções para problemas em conjunto. É importante contextualizar o surgimento das comunidades de prática com o crescimento da internet, pois a atuação em comunidades é considerada como cerne da rede mundial de computadores (MELO; SATTAMINI, 2004; HAUSER; TELLIS; GRIFFIN, 2006).

Cabe destacar que os mecanismos utilizados para o envolvimento de consumidores relatados por Alam (2002), Page e Rosenbaum (1992), Kaulio (1998), Cincianntelli e Magdison (1993), Nambisan e Baron (2007) ocorrem em momentos previamente definidos pela empresa, ou seja, a interação entre consumidores e empresa fica delimitada à programação e aos interesses da empresa. Nesse sentido, Nambisan (2002) discorda de técnicas de envolvimento que sejam pontuais e restritas aos encontros definidos pela empresa. O autor enfatiza que os consumidores podem contribuir em muitas atividades do PDP, desde que os consumidores estejam envolvidos na equipe de trabalho. Entre as atividades, Nambisan (2002) cita a definição da arquitetura do produto, a priorização das funcionalidades do produto a serem ofertadas, a especificação das interfaces disponíveis no produto, a definição dos tamanhos disponíveis, entre outras atividades presentes em todas as fases do PDP. O autor ressalta ainda que, para o envolvimento dos consumidores apresentar resultados satisfatórios, os consumidores devem possuir conhecimentos profundos do produto e também dos recursos tecnológicos 
necessários para participarem efetivamente da equipe de desenvolvimento do novo produto.

Da perspectiva de "market(ing) with" (VARGO; LUSCH, 2004; LUSCH; VARGO, 2006; LUSCH, 2007; PRAHALAD; RAMASWAMY, 2004), a coprodução seria potencializada pela proposta defendida por Nambisan (2002) e por Alam (2002), tendo em vista que esses autores argumentam que o consumidor deve ser compreendido como um membro da equipe de desenvolvimento de novos produtos.

Tendo como base o referencial aqui apresentado, evidencia-se que a proposta de uma nova abordagem para a criação de valor com maior envolvimento do consumidor em processos empresariais implica não apenas mudanças conceituais, mas também a necessidade de um maior entendimento de como tais mudanças são compreendidas pelas organizações e de que forma esse novo modelo afeta ou altera processos organizacionais vinculados à forma tradicional de entrega de valor.

\section{MÉTODO}

A pesquisa do tipo qualitativa foi considerada o método mais adequado para responder à questão de pesquisa deste trabalho. É necessário enfatizar que o envolvimento de consumidores no desenvolvimento de produtos ainda é um fenômeno recente, portanto ainda necessitando que estudos acadêmicos aprofundem o tema proposto.

Para a escolha das empresas pesquisadas, foi considerado como critério básico de seleção a existência de reconhecimento externo por iniciativas de lançamento de produtos, tais como traços de inovação ou constância de lançamentos, observando-se a necessidade de coletar dados que permitissem analisar o fenômeno do envolvimento de consumidores no PDP. A escolha de empresas reconhecidas pelo lançamento constante de novas ofertas no mercado também é alicerçada na literatura, a qual demonstra que empresas com melhor desempenho no lançamento de produtos possuem processos de desenvolvimento mais bem estruturados (COOPER; EDGETT, 1996).

Tendo tal constatação em mente, as empresas participantes do estudo foram selecionadas a partir do conjunto de empresas de bens de consumo da Região Sul que foram agraciadas com prêmios voltados ao reconhecimento de esforços de inovação e/ou novos produtos, a saber:

a) Prêmio Finep de Inovação - premiação organizada pela Financiadora de Estudos e Projetos, empresa pública vinculada ao Ministério da Ciência e Tecnologia, que visa reconhecer e incentivar os esforços de inovação tecnológica no país; b) Prêmio Campeãs da Inovação, da revista Amanhã premiação organizada anualmente pela revista Amanhã que avalia as empresas e as instituições participantes nas dimensões de resultados da inovação, estrutura e cultura organizacional, foco do esforço da inovação, tratamento e orientação à inovação, atitude e processo de geração de criatividade e desenvolvimento inicial da inovação.

Diante dos critérios apresentados, foram realizados contatos com empresas que apresentavam potencial de participação no estudo. Dez empresas aceitaram participar da pesquisa, a seguir relacionadas: Calçados Bibi, Coza, Dimed, Florestal Alimentos, Intelbrás, Karsten, Mundial, Siemens, Tramontina e Whirpool.

A coleta de dados primários foi realizada por meio de entrevistas de profundidade com gestores vinculados ao PDP e da observação das estruturas disponíveis para interação com consumidores. Para a realização das entrevistas de profundidade foi solicitado que as empresas indicassem os gestores responsáveis pelos processos relacionados com desenvolvimento de produtos, tendo em vista a necessidade de exploração dos temas "processos de desenvolvimento de produtos" e "envolvimento de consumidores" (uma síntese do perfil dos entrevistados que inclui gênero, formação e cargo encontra-se no Quadro 1, a seguir).

A partir da indicação dos profissionais, as entrevistas foram pré-agendadas e baseadas em um roteiro semiestruturado validado por acadêmicos, no qual foram abordados os tópicos encontrados na revisão da literatura apresentada neste estudo. Durante a entrevista, apresentava-se ao entrevistado uma série de cartões (cada cartão representando um estágio do PDP) que deveriam ser organizados na ordem empregada no PDP da empresa. Uma vez estabelecida a estrutura adotada pela empresa, cada estágio era abordado individualmente, tendo como foco o envolvimento do consumidor em cada etapa e os mecanismos eventualmente utilizados para permitir tal interação.

As entrevistas apresentaram diferentes padrões de tempo, pois algumas empresas não apenas responderam às questões, mas apresentaram suas instalações e estruturas de interação com o consumidor, quando disponíveis. No que tange especificamente às entrevistas, seu tempo médio girou em torno de 2 horas e meia e ela forams gravadas e posteriormente transcritas, resultando em um conjunto aproximado de 20 laudas por entrevista.

Para a análise de dados foi utilizada a análise de conteúdo categórica, tendo em vista sua adequação para a análise de entrevistas e a interpretação de temas com elevada complexidade (BARDIN, 1977). Para isso, buscou-se na 
revisão da literatura a indicação de categorias relevantes para a interpretação dos conteúdos presentes nos dados coletados, sendo definidas as seguintes categorias de análise: mecanismos de envolvimento; estágios do processo de desenvolvimento de produtos e intensidade de envolvimento.

Para garantir a validade e a confiabilidade das categorias apresentadas, foram tomados os seguintes cuidados metodológicos:

a) Utilização de quatro fontes de evidências: entrevistas, documentos sobre o PDP, unidades de produtos lançados pelas empresas participantes nos últimos três anos e observação direta das estruturas disponíveis para a interação dos consumidores.

b) Gravação das entrevistas de profundidade e arquivamento das anotações realizadas nas visitas às empresas.

c) Elaboração e utilização de protocolo de pesquisa e roteiros semiestruturados para as entrevistas de profundidade e observação direta.

d) Validação do roteiro semiestruturado por especialistas acadêmicos da área de Marketing.

e) Indicação, por parte da empresa, de produtos desenvolvidos com o envolvimento de consumidores.

f) Apresentação dos trechos das entrevistas realizadas com o objetivo de evidenciar os resultados de pesquisa.

\section{RESULTADOS}

Os resultados do estudo estão divididos em três momentos: identificação dos mecanismos de envolvimento; caracterização dos estágios do PDP em que se dá o envolvimento de consumidores; e análise da intensidade desse envolvimento.

\section{Mecanismos de envolvimento}

Estudando-se com profundidade o modo como ocorre o envolvimento de consumidores no processo de desenvolvimento de produtos, verificou-se que as empresas organizam diferentes arranjos ou mecanismos de envolvimento. Foi possível observar, por exemplo, empresas que sequer implantaram técnicas de interação convencionais, como a disponibilidade de um call center. No outro extremo, identificaram-se ambientes como laboratórios de simulação de uso de produtos, propícios para a coprodução de valor, assim como sugeriram Prahalad e Ramaswamy (2003) ao destacarem a relevância da criação de espaços qualificados para o envolvimento de consumidores. Observa-se, assim, um primeiro resultado relevante: ainda que todas as empresas pesquisadas apresentassem traços semelhantes em relação à inovação ou ao constante lançamento de novos produtos, o envolvimento de consumidores no PDP foi bastante variado entre as dez empresas entrevistadas.

Dentro desses diferentes contextos empresariais, foi possível identificar a utilização dos seguintes mecanismos de envolvimento de consumidores: recebimento de telefonemas, faxes, $e$-mails e cartas com sugestões de novos produtos; grupos focais; teste de conceito; teste de produto; visitas e reuniões dos consumidores com a equipe de desenvolvimento de produtos; observação de consumidores; comunidades virtuais (fóruns de discussão na internet) e ferramentas virtuais que permitem a usuários criar design e novas funcionalidades (Quadro 2).

Observa-se que os mecanismos mais interativos (baseados em contatos face a face ou pela internet) são os menos utilizados pelas empresas, havendo maior uso de canais passivos (como telefone, e-mail) e de pesquisas com o emprego de métodos mais tradicionais. Apenas

Quadro 1 - Características dos entrevistados

IDENTIFICACÃO

Entrevistado 1

Entrevistado 2

Entrevistado 3

Entrevistado 4

Entrevistado 5

Entrevistado 6

Entrevistado 7

Entrevistado 8

Entrevistado 9

Entrevistado 10

\section{PERFIL RESUMIDO}

Feminino, engenheira de alimentos, coordenadora de pesquisa e desenvolvimento.

Masculino, designer, gerente de novos produtos.

Masculino, administrador de empresas, coordenador de pesquisa e desenvolvimento.

Masculino, engenheiro elétrico, gerente de desenvolvimento de produtos de comunicação.

Masculino, engenheiro mecânico, gerente de inovação tecnológica.

Masculino, analista de sistemas, gerente de desenvolvimento de novos negócios e inovação.

Masculino, administrador de empresas, diretor de inovação.

Masculino, administrador de empresas, coordenador de desenvolvimento.

Feminino, administradora de empresas, coordenadora de desenvolvimento de novos produtos.

Feminino, engenheira civil, diretora de desenvolvimento de produtos. 
duas empresas utilizam a quase totalidade dos mecanismos questionados, sendo elas empresas com fortes evidências de geração de valor compartilhado.

Adicionalmente, verificou-se que algumas empresas envolvem os seus consumidores de forma passiva, ou seja, oferecem infraestrutura tecnológica para receber sugestões, reclamações e ideias de novos produtos. Dessa forma, o processo de envolvimento ocorre da seguinte maneira: as empresas oferecem a infraestrutura de recebimento de sugestões, os consumidores "depositam" as suas informações, as empresas analisam a pertinência das ideias de novos produtos ou de melhorias em produtos existentes, e, por fim, a partir das ideias selecionadas, inicia-se o processo de desenvolvimento de produtos. É importante salientar que nas empresas que utilizam mecanismos passivos de interação não foi possível reconhecer a prática de diálogo, configurando a empresa apenas como um agente receptivo de informações. Dessa forma, pode-se observar que essas empresas possuem uma sistemática planejada de selecionar e envolver seus consumidores no PDP. No trecho apresentado a seguir, o entrevistado explicita como os consumidores interagem com os mecanismos de envolvimento passivo ofertados pelas empresas.

Existia também uma demanda de consumidores que mandam por e-mail no SAC, entram no site, ligam para cá e dizem: por que vocês não lançam chocolate amargo? O que também acontece algumas vezes, pelo fato da empresa estar há muito tempo no mercado, é recebermos e-mails de consumidores pedindo para relançar antigos produtos. (Entrevistada 1)
Por outro lado, foi possível identificar empresas que organizam a interação de modo ativo, ou seja, a empresa seleciona consumidores para uma participação ativa no PDP a partir de diferentes mecanismos de interação.

\begin{abstract}
Nós realizamos reuniões com grupo de consumidoras, de diferentes classes sociais e também de diferentes estilos de vida. Temos como consumidoras dos nossos produtos desde as clássicas donas de casa até mulheres que possuem uma vida profissional mais agitada. Também fazemos reuniões com chefs de cozinha, sempre com objetivo de testar a usabilidade do nosso portfólio de produtos, assim como os produtos que ainda estão em desenvolvimento. Para isso, criamos dois laboratórios, um da marca A e outro da marca B. São cozinhas montadas que permitem a simulação de utilização dos produtos, e também equipamos com filmadoras, gravadoras de áudio e salão de espelhos. (Entrevistado 5)
\end{abstract}

O exemplo acima demonstra a ocorrência de empresas com avançados processos de envolvimento de consumidores no PDP. Porém, via de regra, observou-se que os mecanismos mais utilizados aproximam-se das técnicas tradicionais de pesquisa de marketing, como grupos focais, pesquisas do tipo survey, observação e entrevistas de profundidade. É importante ressaltar que nesses mecanismos o consumidor atua como um recurso externo, ou seja, a empresa busca respostas pontuais junto ao consumidor, não caracterizando um efetivo e contínuo diálogo entre empresa e consumidores. Por fim, é preciso destacar que, nessas situações, o papel de protagonista da interação cabe à empresa e não aos seus consumidores.

\section{Quadro 2 - Mecanismos de interação/envolvimento consumidor-empresa}

\begin{tabular}{|c|c|c|c|c|c|c|c|c|c|c|}
\hline \multirow[t]{2}{*}{ MECANISMOS DE INTERAÇÃO } & \multicolumn{10}{|c|}{ EMPRESAS } \\
\hline & 1 & 2 & 3 & 4 & 5 & 6 & 7 & 8 & 9 & 10 \\
\hline Interação no ponto de venda & $X$ & $X$ & $X$ & $X$ & $X$ & $X$ & $X$ & $X$ & $X$ & $X$ \\
\hline $\begin{array}{l}\text { Recebimento de telefonemas, faxes, e-mails e cartas com sugestões de } \\
\text { novos produtos }\end{array}$ & $X$ & $x$ & $X$ & $x$ & $X$ & $X$ & $X$ & & & $X$ \\
\hline Grupos focais & $x$ & $x$ & $x$ & $x$ & $x$ & $x$ & $x$ & & & $x$ \\
\hline Teste de produto & $X$ & $X$ & $\mathrm{X}$ & $x$ & $X$ & $X$ & & & $X$ & $X$ \\
\hline Observação de consumidores & $x$ & $x$ & $x$ & $x$ & $x$ & $x$ & $x$ & & & $x$ \\
\hline Visitas e reuniões dos consumidores com a equipe de desenvolvimento de produto & & & & & $\mathrm{X}$ & & & & & \\
\hline Ferramentas virtuais que permitem a usuários criar design e novas funcionalidades & & $x$ & & & & & & & & \\
\hline Comunidades virtuais: fóruns de discussão na internet & & $X$ & & & $x$ & & & & & $X$ \\
\hline
\end{tabular}

Fonte: Coleta de dados. 
Ao explorar os contextos empresariais das organizações que participaram deste estudo, é visível que os gestores do PDP conseguem vislumbrar as contribuições dos consumidores por meio da utilização do chamado mecanismo de representação. Porém, desconhecem como operacionalizá-lo e temem expor a realidade da empresa de forma demasiada. A representação, citada por Alam (2002), caracteriza-se pela participação do consumidor como membro da equipe de desenvolvimento de produto, sendo considerada a forma de maior intensidade de envolvimento de consumidores no PDP. O relato a seguir exemplifica tal contexto:

Parece interessante para a empresa, mas não sei se os consumidores aceitariam um envolvimento tão intenso. Também não sei como funciona em relação às leis trabalhistas, se ele participa todo tempo do desenvolvimento pode requerer alguma coisa? Não sei como seria. Também acho que a empresa necessita tomar alguns cuidados para não se expor demais. (Entrevistado 3)

Nesse caso em particular, observa-se um possível paradoxo no que tange ao envolvimento real do consumidor no desenvolvimento de produtos. De um lado, as empresas pesquisadas destacam a relevância de uma maior interação com os consumidores; porém, de outra parte, assumem uma postura defensiva que mantém os consumidores ao que se pode caracterizar como uma "distância segura" em relação à empresa. Tal contexto aponta para a natural relação entre o envolvimento do consumidor no PDP e os objetivos da organização, uma vez que algumas empresas justificaram a não utilização do mecanismo de representação argumentando que a consulta sistemática pontual em diferentes etapas do PDP, por meio de técnicas mais tradicionais, era suficiente para alcançar os objetivos propostos pela empresa. Nessas situações, é pertinente questionar o quanto os reais propósitos das empresas em disponibilizar mecanismos de interação focalizam o desenvolvimento efetivo de produtos ou apenas um objetivo secundário de criar uma percepção de envolvimento, colaboração junto ao consumidor, porém dentro de limites estritamente definidos pela organização. Na citação apresentada a seguir é possível analisar essa argumentação.

A gente ainda não atuou dessa forma [se referindo ao mecanismo de representação]... mas com os consumidores palpitando, dando ideias no site ou através de e-mails, mais os grupos focais e testes de campo... São formas da gente envolver os consumidores, de entender o que eles valorizam e buscar atributos para outros lançamentos. Já é uma relação bem próxima. (Entrevistado 2)
A partir da identificação dos mecanismos utilizados para envolvimento dos consumidores, foi possível relacionálos com os estágios do PDP.

\section{Estágios do processo de desenvolvimento de produtos (PDP)}

Foram pesquisados os tipos de processos de desenvolvimento de produtos utilizados pelas empresas participantes. Conforme McCarthy e outros (2006) e Cooper, Edgett e Kleinschmidt (2002), os tipos de processos de desenvolvimento de produtos podem ser lineares, recursivos e caóticos. Diante das características de cada processo presentes na literatura e de sua comparação com os dados coletados nas empresas, verificou-se a existência dos três tipos de processos. $\mathrm{O}$ mais presente nas empresas pesquisadas foi o tipo recursivo, sobretudo em função da necessidade de agilidade no lançamento de novos produtos decorrente da competitividade do segmento de bens de consumo.

Ainda com relação aos estágios do PDP, constatou-se que as empresas envolvem os consumidores principalmente nos estágios de geração de ideias, análise da ideia, testes de produto e comercialização (conforme o Quadro 3, a seguir). Embora Alam (2002) defenda a possibilidade de as empresas utilizarem os mecanismos de envolvimento de consumidores em todos os estágios do PDP, não foi possível verificar tal realidade, tendo em vista que os entrevistados rejeitaram completamente o envolvimento de consumidores em estágios como elaboração do business plan, formação da equipe multifuncional e definição da estratégia (estágios esses mencionados por Alam, 2002).

\section{Intensidade do envolvimento dos consumidores}

A partir da identificação dos mecanismos de envolvimento e dos estágios do processo de desenvolvimento, verificouse que as empresas apresentam níveis de intensidade de envolvimento bastante distintos. Diante disso, procurouse relacionar os diferentes níveis de envolvimento que as empresas apresentam com as perspectivas de marketing citadas na literatura da área. Conforme foi apresentado na Fundamentação Teórica, Lusch (2007) dividiu o marketing em três perspectivas: "to market" - ênfase na distribuição; "market(ing) to" - ênfase nos processos de marketing e na satisfação dos consumidores; e por último; "market(ing) with" - ênfase na coprodução de valor.

É possível identificar empresas cujas ações enfatizam características associadas à coprodução de valor, proporcionando espaços interativos e experienciais para seus consumidores, seja em ambientes físicos (como laboratórios, lojas ou ações de ponto de venda) ou ambientes virtuais (como funcionalidades específicas em websites). 
Nesses espaços, os consumidores interagem não apenas com a empresa e seus profissionais, mas com outros consumidores e, eventualmente, profissionais de outras áreas com impacto indireto na geração de valor. Algumas declarações podem ser destacadas neste sentido.

A questão das nossas lojas, hoje, é um ponto muito importante de contato com o consumidor. Estamos começando um projeto de lojas próprias da marca para nos aproximarmos dos consumidores, e elaborarmos uma experiência com a marca mais qualificada. No nosso projeto de varejo, as crianças podem brincar dentro da loja com os nossos produtos e brinquedos. (Entrevistado 2)

Eu monto um ambiente para esse consumidor e coloco as pessoas juntas; não só os consumidores e o pessoal de marketing, mas a pessoa de manufatura e a de tecnologia. Depois, eu os coloco para fazer uma atividade... estão trabalhando, mas não parece. E quando eu faço isso eu também passo por outros conceitos sobre como as pessoas utilizam os produtos e como elas querem utilizar. (Entrevistado 5)

Partindo-se das três perspectivas defendidas por Lusch (2007) e da identificação dos mecanismos de envolvimento de consumidores, as empresas participantes do estudo podem ser divididas em três grupos distintos, da seguinte forma:

a) Empresas com baixa intensidade de envolvimento de consumidores no PDP: o envolvimento fica restrito ao estágio de comercialização. A geração de ideias ocorre por meio de pesquisa em outros mercados e em empresas concorrentes. A empresa não realiza parcerias com outras organizações para realizar o desenvolvimento de produtos. A comunicação da empresa ocorre de forma unilateral, partindo da empresa e chegando até aos consumidores, principalmente no momento da comercialização e na publicidade contratada pela empresa.

b) Empresas com moderada intensidade de envolvimento de consumidores no PDP: o envolvimento de consumidores fica concentrado nos estágios de geração de ideias, análise da ideia e teste de produto por meio da utilização de técnicas tradicionais de pesquisa de marketing, como grupos focais e observações. A comunicação entre empresa e consumidores não é reconhecida pelo diálogo, ocorrendo de forma unilateral, com os consumidores fornecendo informações por meio das pesquisas de marketing e as empresas se comunicando por meio da sua propaganda/publicidade.

c) Empresas com alta intensidade de envolvimento de consumidores no PDP: a geração de ideias é compartilhada entre equipe de desenvolvimento, consumidores, fornecedores e outros atores. São utilizados diferentes tipos de mecanismos de envolvimento com consumidores, desde técnicas tradicionais de pesquisa de marketing até reuniões com grupos de consumidores e ferramentas virtuais de interação. A comunicação é bidirecional, consumidores e representantes das empresas dialogam. As empresas investem uma infraestrutura dedicada ao envolvimento de consumidores, como laboratórios de testes de produtos, dispositivos na internet, pontos de venda interativos etc.

Quadro 3 - Mecanismos de envolvimento de consumidores e etapas do PDP

\begin{tabular}{|l|l|}
\hline \multicolumn{1}{|c|}{ MECANISMOS DE ENVOLVIMENTO } & \multicolumn{1}{c|}{ ETAPAS DO PDP } \\
\hline $\begin{array}{l}\text { Recebimento de telefonemas, faxes, e-mails e cartas com } \\
\text { sugestões de novos produtos }\end{array}$ & Geração de ideias, Comercialização \\
\hline $\begin{array}{l}\text { Grupos focais } \\
\text { Teste de conceito }\end{array}$ & $\begin{array}{l}\text { Geração de ideias, Análise da ideia, Teste de protótipo de } \\
\text { produto, Comercialização, Teste de mercado }\end{array}$ \\
\hline Teste de produto & Análise da ideia \\
\hline $\begin{array}{l}\text { Visitas e reuniões dos consumidores com a equipe de } \\
\text { desenvolvimento de produto }\end{array}$ & Teste de protótipo de produto \\
\hline Observação de consumidores & $\begin{array}{l}\text { Geração de ideias, Análise da ideia, Teste de protótipo de } \\
\text { produto, Comercialização }\end{array}$ \\
\hline Comunidades virtuais: Fóruns de discussão na internet & $\begin{array}{l}\text { Geração de ideias, Análise da ideia, Desenvolvimento de } \\
\text { produtos, Comercialização }\end{array}$ \\
\hline $\begin{array}{l}\text { Ferramentas virtuais que permitem a usuários criar design } \\
\text { e novas funcionalidades }\end{array}$ & Geração de ideias \\
\hline
\end{tabular}

Fonte: Coleta de dados. 


\section{CONCLUSÕES}

Ao concluir este estudo, é necessário retomar a discussão apresentada no referencial teórico sobre o envolvimento de consumidores em uma perspectiva mais ampla dos processos de marketing, assim como defendem Vargo e Lusch (2004), Lusch e Vargo (2006), Lusch (2007), Prahalad e Ramaswamy (2004), Grönroos (2006), Ballantyne e Varey (2006) e Penaloza e Venkatesh (2006).

No referencial teórico, foi possível compreender que os estudiosos identificados com a perspectiva "market(ing) with" defendem que o marketing deveria evoluir para um novo patamar, representado pela interação e pela participação de consumidores nos processos de marketing, ou seja, pela coprodução de valor. Adicionalmente, verificouse na coleta e na análise dos dados desta pesquisa que as empresas participantes caminham para práticas de maior envolvimento com consumidores no PDP. No entanto, constatou-se que, mesmo nas empresas que foram classificadas no nível de maior intensidade de envolvimento, os processos ainda estão em estágio embrionário. Algumas dúvidas manifestadas pelos entrevistados resultam em barreiras para uma maior inserção dos consumidores no PDP. Pode-se mencionar, por exemplo, questionamentos sobre os meios de operacionalizar tais mecanismos; a existência de temores quanto ao vazamento de informações; preocupações com questões trabalhistas ou com a imagem da empresa, e custos adicionais no processo. Dessa forma, evidenciam-se algumas lacunas ainda existentes entre o conceito de coprodução de valor e as práticas de marketing nas empresas pesquisadas.

Nesse mesmo contexto, outra questão relevante trata da compreensão e dos propósitos das empresas em envolver seus consumidores no PDP. Algumas das informações coletadas demonstram que as oportunidades de interação em si podem ser o principal objetivo buscado pelas organizações, ou seja, mecanismos que geram a percepção por parte do consumidor de que ele está envolvido ou colaborando com a empresa - em vez de focar realmente direcionado ao desenvolvimento do produto. Nesse sentido, as empresas parecem gerenciar a interação com o consumidor dentro de limites bastante restritos, ignorando alguns pressupostos já destacados por Prahalad e Ramaswamy (2004) em seu modelo DART, o qual preconiza o diálogo, o acesso, a transparência nas informações e o compartilhamento do risco como pilares para a criação compartilhada de valor.

Como principal conclusão do estudo, verificou-se que o envolvimento de consumidores ocorre de diferentes formas e em diferente intensidade, mesmo considerando um grupo de empresas razoavelmente homogêneo em termos de maturidade e inovação no desenvolvimento de novos produtos. Isso aponta para a necessária investigação dos elementos que viabilizam ou possibilitam a adoção de ações voltadas à coprodução de valor. Fatores como a cultura organizacional, a natureza do produto, as características do mercado e as próprias competências da empresa representam potenciais elementos que interferem no efetivo engajamento das organizações em ações de coprodução e, em particular, de consumidores no PDP.

Observou-se, também, uma tendência ou desejo de as empresas buscarem formas mais evoluídas de interação com os consumidores, porém as práticas gerenciais relacionadas aos mecanismos de envolvimento ainda estão em processo de amadurecimento, sendo que a maioria das empresas pesquisadas emprega prioritariamente técnicas tradicionais de pesquisa de marketing.

Um dos aspectos relevantes do estudo foi a possibilidade de caracterização de elementos centrais associados com o envolvimento de consumidores no PDP. Partindo-se de três níveis de envolvimento (baixo, moderado e alto), o Quadro 4 sintetiza tais características.

Mesmo tendo sido realizado dentro dos parâmetros científicos recomendados para estudos de natureza qualitativa, o trabalho aqui apresentado possui algumas limitações, que devem ser detalhadas para melhor compreensão de seus resultados e implicações.

Uma limitação inicial refere-se à extensão dos resultados aqui encontrados. Tendo em vista as características do estudo, acredita-se que as conclusões aqui apresentadas estabeleçam uma orientação sólida para futuros estudos na área; contudo, sem determinar possíveis relações entre as diversas variáveis que compõem a decisão das empresas em envolver ou não seus consumidores no PDP. Adicionalmente, o fato de a pesquisa ter sido realizada em empresas da Região Sul do Brasil pode implicar a presença de traços culturais específicos dos executivos em atuação nessa região geográfica.

Por fim, diante da necessidade de definir critérios mínimos para a seleção das empresas participantes, optou-se pelo uso do reconhecimento externo à inovação e lançamento de novos produtos por parte das empresas. Tal critério, contudo, não pode ser considerado um parâmetro conclusivo no que tange ao envolvimento de consumidores do PDP. Empresas não premiadas ou que simplesmente não participam dos processos de premiação aqui utilizados podem naturalmente contemplar mecanismos de envolvimento e interação com consumidores. Além disso, a partir do estudo não é possível determinar o quanto ou quais empresas eventualmente estruturaram processos de envolvimento dos consumidores com o intuito mais 
restrito de atender a requisitos dos próprios processos de premiação. Em que pesem tais limitações, cabe mencionar que os resultados do estudo apontam para diferentes posturas empresariais em relação ao envolvimento de consumidores do PDP, sinalizando que o engajamento em processos competitivos (premiações) não determina necessariamente novos arranjos no desenvolvimento de produtos. Para futuros trabalhos, porém, a definição de um conjunto mais detalhado de critérios passíveis de análise prévia por parte do pesquisador é recomendável.

Diante dos achados desta pesquisa, realizada junto a relevantes empresas de bens de consumo da Região Sul do Brasil, é possível concluir que o tema envolvimento de consumidores no PDP e a própria coprodução de valor representam um campo fértil para novos estudos acadêmicos.

Constatou-se que algumas relações do envolvimento de consumidores precisam ser mais bem exploradas em futuros estudos. Há indícios de que tanto fatores internos (como cultura, competências organizacionais e características do produto) quanto externos (como as características do mercado, consumidores e concorrentes) influenciam a viabilidade e o interesse das empresas na implantação de processos de coprodução de valor. Compreender adequadamente quais são e como tais elementos atuam representa uma contribuição futura relevante para o entendimento do próprio conceito de cocriação de valor.

Por outro lado, a identificação das consequências de um maior envolvimento do consumidor no PDP - e em outros processos da empresa - constitui-se em outra ampla via para futuros estudos. Maiores níveis de envolvimento resultam em melhor desempenho no lançamento de novos produtos? Que resultados são observados a partir de uma maior interação entre consumidores e empresas no que tange à aprendizagem e a benefícios mútuos? Adicionalmente, estudos para a compreensão das relações entre experiências de consumo e envolvimento de consumidores contribuiriam para uma delimitação mais segura dos constructos discutidos no trabalho. Por fim recomenda-se a realização de estudos quantitativos que possam contribuir com a caracterização de níveis de intensidade de envolvimento sugerida neste trabalho.

\section{NOTA DA REDAÇÃo}

Artigo originalmente apresentado no EnANPAD 2009 e submetido à RAE em regime de fast-track.

\section{REFERÊNCIAS}

ALAM, I. An exploratory investigation of user involvement in new service development. Journal of the Academy of Marketing Science, v. 30, n. 3, p 250-261, 2002

ALAM, I; PERRY, C. Customer-oriented new service development process. The Journal of Services Marketing, v. 16, n. 6, p. 515-534, 2002

BALLANTYNE, D; VAREY, R. Creating value-in-use through marketing interaction: the exchange logic of relating communicating and knowing. Marketing Theory, v. 6, n. 3, 2006

\section{Quadro 4 - Caracterização das empresas e os níveis de envolvimento de consumidores no PDP}

BAIXO ENVOLVIMENTO DE CONSUMIDORES NO PDP

\section{MODERADO ENVOLVIMENTO DE} CONSUMIDORES NO PDP
ALTO ENVOLVIMENTO DE

CONSUMIDORES NO PDP
As ideias de novos produtos são normal- A geração de ideias é realizada com a coleta mente geradas a partir de produtos existentes, disponíveis em outros mercados ou de empresas concorrentes.

A comunicação é restrita ao contato no ponto de venda.

A infraestrutura disponível para envolvimento de consumidores está restrita ao ponto de venda.

Não tem foco na experiência do consumidor. de informações por meio de técnicas tradicionais de pesquisa de marketing.

A comunicação é unidirecional. 0 consumidor fornece informações para a empresa.

A infraestrutura utilizada para o envolvimento de consumidores está na contratação de empresas de pesquisa de marketing.
A geração de ideias é compartilhada entre equipe de desenvolvimento, consumidores, fornecedores, entre outros atores.

A comunicação é bidirecional. Consumidores e representantes da empresa dialogam.

A empresa investe uma infraestrutura dedicada para o envolvimento de consumidores como laboratórios de testes, dispositivos na internet, PDV interativo.

Realização de ações que valorizam a experiência dos consumidores com a empresa. zam a experiência dos consumidores com a empresa/produto.

Fonte: Coleta de dados. 
BARDIN, L. Análise de conteúdo. Lisboa: Edições 70, 1979.

CHRISTENSEN, C. M; BOWER, J. L. Customer power, strategic investment, and failure of leading firms. Strategic Management Journal, v. 17, n. 3, p. 197-218, 1996.

CHRISTENSEN, C. M. Making strategy: learning by doing. Harvard Business Review, v. 75, n. 6, p. . 1997.

CICIANNTELLI, S; MAGDISON, J. Consumer idealized design: involving consumers in the product development process. Journal of Product Innovation Management, v. 10, n. 4, p. 341-347, 1993.

CONNER, K. R. A historical comparison of resource-based theory and five schools of thought within industrial organization economics: do we have a new theory of the firm? Journal of Management, v. 17, n. 1, p. 121154, 1991

DAY, G. S; WENSLEY, R. Assessing advantage: a framework for diagnosing competitive superiority. Journal of Marketing, v. 52, n. 2, p. 1- 20, 1988.

DESHPANDÉ, R; FARLEY, J. U; WEBSTER JR, F. E. corporate culture customer orientation, and innovativeness in Japanese firms: a quadrad analysis. Journal of Marketing, v. 57, n. 1, p. 23-37, 1993.

ENGEL, J. F; BLACKWELL, R. D; MINIARD, P. W. Comportamento do consumidor. 8. ed. Rio de Janeiro: LTC, 2000.

ENGEL, J. F. Risk taking and information handling in consumer behavior. Journal of Marketing, v. 32, n. 3, p. 24-29, 1968.

FRANKE, N; VON HIPPEL, E; SCHREIER, M. Finding commercially attractive user innovations: a test of lead-user theory. The Journal of Product Innovation Management, v. 23, n. 4, p. 301-315, 2006.

GALLOUJ, F; WEINSTEIN, O. Innovation in services. Research Policy, v. 26, p. 537-556, 1997.

GRIFFIN, A; HAUSER, J. R. The voice of the customer. Marketing Science, v. 12, n. 1, p. 1-27, 1993.

HAMEL, G; PRAHALAD, C. K. Competindo pelo futuro: estratégias inovadoras para obter o controle do seu setor e criar mercados de amanhã. 8 . ed. Rio de Janeiro: Campus, 1995.

HAUSER, J; TELLIS, G. J; GRIFFIN, A. Research on innovation: a review and agenda for marketing science. Marketing Science, v. 25, n. 6, 2006.

HOWARD, J. A; SHETH, J. N. The theory of buyer behaviour. British Journal of Marketing, v. 4, n. 2, 1970.

JAWORSKI, B; KOHLI, A. Market orientation: antecedents and consequences. Journal of Marketing; v. 57, n. 3, 1993.

JONES, D. Simon Litman (1873-1965): pioneer marketing scholar. Marketing Theory, v. 4, n. 4, p. 343-361, 2004.

KAULIO, M. A. Customer, consumer and user involvement in product development: a framework and a review of selected methods. Total Quality Management, v. 9, n. 1, 1998.

KUMAR, V; PETERSEN, J. A. Using a customer-level marketing strategy to enhance firm performance: a review of theoretical and empirical evidence. Journal of the Academy of Marketing Science, v. 33, n. 4, 2005.

LEONARD-BARTON, D. Managing creative abrasion in the workplace. Harvard Business Review, v. 73, n. 4, p. , 1995.

LILIEN, G. L. e outros. Performance assessment of the lead user ideageneration process for new product development. Management Science, v. 48, n. 8 , p. 2002.
LUSCH, R. F. Marketing's envolving identify: defining our future. American Marketing Association, v. 26, n. 2, 2007.

LUSCH, R. F; VARGO, S. L. Service - dominant logic: reactions, reflections and refinements. Marketing Theory, v. 6, n. 3, 2006.

MAKLAN, S; KNOX, S; LYNETTE, R. News trends in innovation and customer relationship management. International Journal of Market Research, v. 50, n. 2, 2008.

MCCARTHY, I. P; TSINOPOULOS, C; ALLEN, P; ROSE-ANDERSSEN, C. New product development as a complex adaptive system of decisions. Journal of Product Innovation Management, v. 23, n. 5, p. 437-456, 2006.

MÖLLER, K; RAJALA, R; WESTERLUND, M. Service innovation myopia? California Manageament Rewiew, v. 50, n. 3, 2008.

NAMBISAN, S. Designing virtual customer environments for new product development: toward a theory. Academy of Management Review, v. 27, n. 3, 2002.

NAMBISAN, S; BARON, R. A. Interactions in virtual customer environments: implications for product support and customer relationship management. Journal of Interactive Marketing, v. 21, n. 2, 2007.

NARVER, J. C; SLATER, S. F. The effect of marketing orientation on business profitability. Journal of Marketing, v. 54, n. 4, 1990.

PAGE, A. L; ROSENBAUM, H. F. Developing an effective concept testing program for consumer durables. Journal of Product Innovation Management, v. 9, n. 4, 1992.

PRAHALAD, C. K; RAMASWAMY, V. The new frontier of experience innovation. MIT Sloan Management Review, v. 44, n. 4, p. 12-18, 2003.

PRAHALAD, C. K; RAMASWAMY, V. Co-creation experiences: the next practice in value creation. Journal of Interactive Marketing, v. 18, n. 3, 2004

ROTHWELL, R. Towards the fifth-generation innovation process. International Marketing Review, v. 11, n. 1, p. 7-32, 1994.

SCARABOTO, D. Comunidades on-line como fonte de informação em marketing: reflexões sobre possibilidades e práticas. Revista Eptic On Line, v. 8, n. 3, 2006.

SHAW, E. H; JONES, D. G. B. A history of schools of marketing thought. Marketing Theory, v. 5, n. 3, p. 239-281, 2005.

SHETH, J. N; SISODIA, R. S; SHARMA, A. The antecedents and consequences of customer-centric marketing. Journal of the Academy of Marketing Science, v. 28, n. 1, 2000.

SLATER, S. F; NARVER, J. C. Market orientation and the learning organization. Journal of Marketing, v. 59, n. 3, 1995.

URBAN, G. I; VON HIPPEL, E. Lead user analyses for the development of new industrial products. Management Science, v. 34, n. 5, 1988.

VARGO, S. L; LUSCH, R. F. Evolving to a new dominant logic for marketing. Journal of Marketing, v. 68, n. 1, p. 1-17, 2004.

VON HIPPEL, E. Implications for innovation. Management Science, v. 40, n. 4, 1994.

VON HIPPEL, E. Lead users: a source of novel product concepts. Management Science, v. 32, n. 7, p. 1986.

VON HIPPEL, E. User toolkits for innovation. Journal of Product Innovation Management, v. 18, n. 3, p., 2001. 